\title{
Visceral obesity alters expression of inflammatory mediators in patients undergoing gastrointestinal resection
}

\author{
S. L. Doyle, C. L. Donohoe, J. V. Reynolds, G. P. Pidgeon and J. Lysaght \\ Department of Surgery, Trinity College Dublin/St James' Hospital, Dublin 8, Republic of Ireland
}

\begin{abstract}
Visceral adipose tissue fuels a state of chronic inflammation ${ }^{(1)}$ and has been identified as a risk factor for post-op complications ${ }^{(2)}$. In this study we first aimed to retrospectively investigate the association between visceral obesity and post-op inflammation using the markers CRP and CRP:albumin ratio. Secondly, we aimed to identify additional inflammatory mediators that are differentially regulated in viscerally obese patients post-op using prospectively recruited patients.

Retrospectively, visceral fat area (VFA) was calculated from diagnostic CT scans of gastrointestinal cancer patients undergoing resection $(n=324)$. Visceral obesity was classified as a VFA $>130 \mathrm{~cm}^{2(3)}$. CRP and albumin levels pre-op and at days $1,3,7$ and 14 postop were obtained from the laboratory database. As part of ongoing prospective studies, peripheral blood mononuclear cells (PBMC), serum and plasma samples are collected on oesophageal adenocarcinoma patients pre-op and on days 1, 3, 7 and 14 post-op. In a test cohort of 3 non-obese and 3 obese patients, PBMC collected pre-op and on days 1 and 7 post-op were analysed for expression of 370 genes involved in acute inflammatory response using a PCR based array. Circulating levels of TNF- $\alpha$, IL- 6 , IL- 8 and IFN- $\gamma$ were measured by ELISA in 12 non-obese and 16 obese patients at all time points. In all cases $p<0.05$ was considered statistically significant.

Following resection, visceral obesity was significantly associated with higher CRP on days 1, 3, 7 and 14 post-op and higher CRP:albumin ratio on day 3 post-op $(p<0.05)$. Higher circulating levels of TNF- $\alpha$, IL-6, IL- 8 and IFN- $\gamma$ were observed in obese compared to non-obese patients on day 1 post-op but these differences were not significant. The inflammatory array revealed an upregulation on post-op day 1 in IL-6 expression (>7-fold) in PBMC from obese compared to non-obese patients. In addition, members of the TNF super family, TNFSF14 and lymphotoxin- $\gamma$, which induce inflammatory cytokine production and NFkB activation, were upregulated in obese ( $>5$-fold and $>2$-fold respectively) compared to non-obese patients.

Excess visceral adiposity significantly alters the acute inflammatory response post-op. Inflammatory mediators such as IL-6, TNFSF14 and lymphotoxin- $\beta$ may prove important targets for pharmacotherapy and immunonutrition to improve post-op outcomes in obese patients.
\end{abstract}

1. Kershaw EE \& Flier JS (2004) J Clin Endocrinol Metab 89, 2548-2556.

2. Doyle SL, Lysaght J \& Reynolds JV (2010) Obes Rev 12, 875-886.

3. Despres J-P \& Lamarche B (1993) Nutr Res Rev 6, 137-159. 